\title{
Benchmarking EUV Mask Inspection Beyond 0.25 NA
}

\author{
K. A. Goldberg ${ }^{1}$, P. Naulleau ${ }^{1}$, I. Mochi ${ }^{1}$, E. H. Anderson ${ }^{1}$, S. B. Rekawa ${ }^{1}$, C. D. Kemp ${ }^{1}$, \\ R. F. Gunion ${ }^{1}$, H.-S. Han ${ }^{2}$, S. Huh ${ }^{3}$ \\ ${ }^{1}$ Mail Stop 2-400, Lawrence Berkeley National Laboratory, Berkeley, CA 94720 USA \\ ${ }^{2}$ Photomask Team, Samsung electronics, Ban-Wol, Hwasung, Kyunggi, Korea 445-701 \\ ${ }^{3}$ SEMATECH, 255 Fuller Road, Suite 309, Albany, NY 12203, USA
}

The SEMATECH Berkeley Actinic Inspection Tool (AIT) is an EUV-wavelength mask inspection microscope designed for direct aerial image measurements, and pre-commercial EUV mask research. Operating on a synchrotron bending magnet beamline, the AIT uses an off-axis Fresnel zoneplate lens to project a high-magnification EUV image directly onto a CCD camera. We present the results of recent system upgrades that have improved the imaging resolution, illumination uniformity, and partial coherence. Benchmarking tests show image contrast above $75 \%$ for 100 -nm mask features, and significant improvements and across the full range of measured sizes. The zoneplate lens has been replaced by an array of user-selectable zoneplates with higher magnification and NA values up to 0.0875 , emulating the spatial resolution of a 0.35-NA $4 \times$ EUV stepper. Illumination uniformity is above $90 \%$ for mask areas $2-\mu \mathrm{m}$-wide and smaller. An angle-scanning mirror reduces the high coherence of the synchrotron beamline light source giving measured $\sigma$ values of approximately 0.125 at 0.0875 NA.

\section{INTRODUCTION}

Mask research is central to the development and commercialization of EUV lithography. The resonant-reflective nature of EUV multilayer coatings, and the wavelength-specific properties of materials used to create EUV masks make it necessary to conduct research at the operational EUV wavelength. Therefore, the development and optimization of EUV mask architectures and defect detection and mitigation technologies currently relies on pre-commercial printing and at-wavelength inspection tools such as the SEMATECH Berkeley Micro-Field Exposure Tool (MET) [1], and the SEMATECH Berkeley Actinic Inspection Tool (AIT) $[2,3,4]$.

\footnotetext{
1 email address: KAGoldberg@1bl.gov
}

Electronic mail: KAGoldberg@lbl.gov 
The AIT is a dual-mode microscope that can operate in scanning-beam and imaging modes. In the scanning-beam mode [4], the EUV beam is focused onto a single point on the mask with a beam diameter of 1 to $5 \mu \mathrm{m}$. The reflected signal, including both bright field specular reflection, and dark field scattered light, is recorded by a three-element photodiode as the mask translates below the focused beam. In imaging mode [3] the AIT operates as an EUV Fresnel zoneplate (holographic lens) microscope: the sub-mm diffractive lens sits above the mask surface and projects an image of the illuminated mask with high magnification, onto an EUV-sensitive CCD camera.

The AIT is now routinely used in studies of pattern defect repair [5], sensitivity to buried, substrate phase defects $[6,7]$, mask contrast, and aerial image properties.

A. System Description. Detailed descriptions of the AIT optical system and performance specifications in imaging mode have been presented previously [2, 3, 4]. The AIT uses a synchrotron bending magnet beamline at the Advanced Light Source, at Lawrence Berkeley National Laboratory (LBNL). A monochromator provides EUV illumination with a tunable wavelength and bandwidth: the AIT typically operates at 13.4-nm wavelength with a bandwidth, $\lambda / \Delta \lambda$, of 1600 . The narrow bandwidth is required to avoid chromatic aberration from the zoneplate lens.

Glancing incidence beamline mirrors, including a Kirkpatrick-Baez (KB) mirror pair create an intermediate focus of approximately $100 \times 300 \mu \mathrm{m}$, which is re-imaged onto the mask by a $20 \times$ demagnification, EUV Schwarzschild objective. Using an off-axis sub-aperture of the Schwarzschild objective, the central ray of the illuminating beam is inclined at $6^{\circ}$ from vertical as it reaches the upward-facing mask. In imaging mode, we typically operate with the mask surface $200-400 \mu \mathrm{m}$ below the Schwarzschild's focal plane to provide a larger, and more uniform illumination area.

In the AIT, an off-axis zoneplate lens with a focal length of $1 \mathrm{~mm}$ or less projects a highly magnified image of the mask surface onto an EUV CCD camera. The illuminating beam passes downward through an un-patterned region of the zoneplate membrane. The zoneplate is 
positioned to collect the reflected light, and focus it to a plane several hundred mm away. The zoneplate's off-axis design provides angular separation of the various diffraction orders so that the focusing first order light does not overlap the other orders in the image plane. A small, $45^{\circ}$, multilayer-coated turning mirror, is positioned between the Schwarzschild housing and the mask, and reflects the light horizontally, toward the CCD.

The AIT routinely records 250-350 high-quality images in numerous through-focus series per eight-hour shift. Typical exposure times range from 0.5 seconds during alignment, to approximately 25 seconds for high-resolution images.

B. 2008 Upgrades. Upgrades conducted in 2008 have made significant improvements to the imaging performance, including resolution and contrast, and illumination uniformity and partial coherence. The most significant additions were a new zoneplate design, a CCD camera with higher pixel density, and an actuator to continuously scan the angle of the horizontal KB mirror. Measurements performed to quantify these improvements are the primary subject of this paper.

In a new zoneplate design, Fig. 1, a single silicon-nitride membrane window in an etched Si wafer was patterned with an array of five zoneplates, each with different optical properties. The zoneplate array allows the operator to select from three different NA values at highest magnification. Lenses with NA values of $0.0625,0.075$, and 0.0875 , emulate the spatial resolution of a $4 \times$ EUV stepper with $0.25,0.30$, and $0.35 \mathrm{NA}$, respectively.

One goal of the new design is to achieve higher pixel density in the recorded images. A new EUV CCD camera was installed with $13.5 \mu$ m pixel width, replacing the $20-\mu \mathrm{m}$-pixel camera used before 2008. The new zoneplates are designed with a 33\% higher magnification ratio. Combining the higher imaging magnification with smaller pixels, the equivalent mask plane pixel density is now $16 \mathrm{~nm}$ per pixel, compared with $31 \mathrm{~nm}$ per pixel in 2007 . The diffraction-limited resolution of the AIT zoneplates is always several times larger than this pixel equivalent size, so the image is adequately sampled. For example, at $0.35-\mathrm{NA}, 4 \times$ equivalent, the true NA of the zoneplate is 0.0875 , the Rayleigh resolution $(0.61 \lambda / \mathrm{NA})$ is $94 \mathrm{~nm}$, and the outer zone width, $\Delta r$, of this $6^{\circ}$ off-axis lens is $35 \mathrm{~nm}$. 
Figure 2 shows three image details of similar darkfield mask features with 100-nm line width, recorded before and after the upgrade, and with the higher-NA zoneplate lens.

To improve the illumination uniformity and reduce the high degree of coherence, the stage that holds the horizontal element of the KB mirror pair was retrofitted with a small, in-vacuum scanning motor that controls the tilt angle. We oscillate the tilt angle during exposures, scanning through a range of illumination angles.

C. Carbon Contamination. Although power levels in the AIT are relatively low, compared with proposed commercial EUV lithography tools_- not more than tens of $\mathrm{nW}$ reach the maskintensive use over a period of months to years leads to the buildup of carbon contamination. Contamination leads to reduced flux levels and non-uniform illumination, discussed in the following section. We take several steps to mitigate carbon contamination: ultra-high vacuum protocols are followed to limit contamination and avoid outgassing materials; only narrow-band EUV illumination is used, to avoid out of band radiation; and a low partial pressure of oxygen gas, less than $10^{-4}$ Torr, flows into the endstation in the vicinity of the $45^{\circ}$ mirror that precedes the Schwarzschild objective.

Despite these measures, we observe the build-up of carbon contamination, and we periodically replace the accessible optical elements in the endstation. Recently, after approximately 240 hours of use, carbon was visible on the zoneplate membrane. The abovementioned $45^{\circ}$ turning mirror, however, showed no visible carbon build up during the same period. Furthermore, we believe that the reflectivity and uniformity of the Schwarzschild objective's pupil have been degraded over time as evidenced by the incident-angle dependence of the mask illumination intensity, and the non-uniformities in the static illumination pattern. That element, however, cannot easily be replaced on cleaned in situ.

\section{ILLUMINATION UNIFORMITY}

Illumination uniformity is a property that affects all measurements made from recorded aerial images. Achieving uniform illumination in the AIT is challenging because of the nature of 
the existing synchrotron-beamline illumination system. The light undergoes nine reflections between the bending magnet source and the mask. The beam is also transmitted through the pattern-free region of a silicon-nitride membrane that contains the zoneplate lenses. Different types of surface contamination, imperfect focusing, and other sources of non-uniformity are difficult to avoid or eliminate completely.

To improve uniformity, we have installed an angle scanning motor onto the stage that supports the horizontal KB mirror. During the high-resolution exposures, small angle changes sweep the illumination pattern in the $x$ direction. The scan rate depends on the amplitude: $1.7 \mathrm{~Hz}$ is a typical frequency, and in a 25 second exposure, the angle scanner completes almost 15 cycles.

A. Illumination uniformity measurement. Using images recorded from a blank EUV mask, we can quantify the measured illumination uniformity as a function of the area of interest, with and without the actuation of the angle-scanning mirror. To perform this measurement, two images close to best focus were selected from through-focus series, and uniformity analysis was performed on sub-domains of the full images. Both images were 25-second exposures, recorded a few minutes apart. One of the images was recorded with the angle-scanning mirror active; and the other, with the mirror in a static, centered position.

Square sub-domains were extracted from near the centers of the images with widths corresponding to the following mask dimensions: $1,2,3,5,8$, and $10 \mu \mathrm{m}$. (The full-width of the $2048 \times 2048$ pixel images corresponds to approximately $32.8 \mu \mathrm{m}$, and the linear scale is approximately $16 \mathrm{~nm}$ per pixel.) For each image and sub-domain size being evaluated, 100 similar sub-domains were extracted and examined: the centers of these overlapping sub-domains were spaced in a 10 by 10 grid with 20 pixel steps.

For this analysis, uniformity is defined as the ratio of the minimum value to the maximum value within each sub-domain. Figure 3 contains the average measured uniformity for the six region sizes, calculated from each image. The standard deviation values for the data points are indicated by the error bar lengths. As expected, smaller regions achieve higher average 
uniformity. Values above $90 \%$ were measured for region widths of $2-\mu \mathrm{m}$ and below. In the static illumination cases, the large standard deviations of the 2,3 , and $5 \mu \mathrm{m}$ sub-domains reveal the much greater variability in neighboring regions that the scanning illumination addresses. The data shows that illumination angle scanning effectively smoothes the non-uniformities that occur in the $2-5 \mu \mathrm{m}$ length scale.

\section{CONTRAST TRANSFER FUNCTION-CTF}

The CTF of an optical system is the ratio of the contrast in the image to that in the object as a function of the feature size. The more familiar modulation transfer function (MTF) is strictly defined for incoherent illumination conditions $(\sigma=\infty)$. The CTF of a high-quality imaging system is a function of its illumination coherence properties, the pupil's shape and numerical aperture, and the incident light wavelength. Coherent or nearly coherently illuminated aberration-free systems maintain high contrast, with a sharp drop near the resolution limit. Aberrations reduce the contrast in a manner that varies with both the direction and size of features being imaged.

A. CTF Measurement. Hundreds of images were recorded to assess the AIT's CTF, using mask line-widths from 100 to 500-nm, and two different elbow pattern orientations. Contrast measurement involves (1) the extraction of elbow pattern regions from each image, (2) intensity normalization across the line region, and (3) the evaluation of the contrast for the central three elbow lines, averaged over several pixels along the length of the line. From the four available pattern directions, the analysis selected the peak contrast at best focus for each measurement series. Note that the object (mask) modulation is slightly less than $100 \%$ (97.4\%), based on the measured light intensity in the pure absorber regions [3].

B. Darkfield CTF before and after upgrades. Dark-field measurements are shown in Fig. 4. A direct comparison of contrast data recorded before and after the upgrades, using similar 0.0625NA zoneplate lenses, shows that a significant increase in contrast has been achieved. Data from the new 0.0875-NA zoneplate are also shown in Fig. 5. Although the higher-NA lens achieves greater contrast for the smallest measured features (above $75 \%$ contrast for $100-n m$ CD), the lower NA lens has slightly higher contrast for larger features. An increased aberration magnitude 
associated with the higher-NA lens may be responsible for the lower contrast. For comparison, the dashed curves in the figure show the calculated ideal CTF performance of two systems: (a) 0.0625-NA and $\left(\sigma_{x}, \sigma_{y}\right)=(0,0.175)$; and (b) $0.0875-\mathrm{NA}$ and $\left(\sigma_{x}, \sigma_{y}\right)=(0,0.125)$.

C. Lower contrast in bright-field. Compared with the dark-field measurements, the data show lower bright-field contrast (typically by several percent). The difference is consistent with the 2$3 \%$ flare magnitude measured in a similar zoneplate lens used in 2007 [3]. In addition, a secondary source of contrast reduction that affected the bright-field measurements was a weak but persistent reflected beam overlap that came from an unobstructed light path reaching the CCD camera. (A new zoneplate membrane design has been created to block this unwanted light.)

The limits of the test mask's quality were not addressed in this study. Dark-field elbows were clearly resolved as small as 100-nm CD. However, bright-field features below 125-nm were not resolved on the mask, and the 125-nm features were irregular.

D. Aberrations. Aberrations in the AIT imaging depend sensitively on the system alignment, including the illumination angles, and the zoneplate position. Even if the zoneplate were aberration-free, displacements and misalignment can introduce aberrations. Furthermore, small variations of the aberrations across the field of view are an unavoidable consequence of using a single lens imaging system. We expect that aberrations caused by misalignment would grow as powers of the NA value, and would therefore impact the high-NA imaging more severely.

When this data was collected, astigmatism was the dominant aberration near the center of the AIT's field of view. Depending on the elbow feature size, astigmatism causes several microns of astigmatic displacement (i.e. the peak contrast for orthogonal pattern directions occurs in focal planes separated by a few microns). Figure D shows through-focus images of a 175-nm square contact through focus in which the presence of astigmatism is clear, and a small coma-like effect is also visible. The detailed quantification of these aberrations is now in progress.

\section{ILLUMINATION COHERENCE}

The bending-magnet beamline source provides a high degree of illumination coherence. The coherence is reduced in the $x$-direction by the action of the angle-scanning mirror, described in 
Section II. Quantifying the illumination coherence properties is essential for imaging data analysis, and for comparison with printing and modeling.

We assess the degree of partial coherence by studying the contrast of dense line features in large, through-focus series [3]. The observed contrast oscillates between high and low values as the mask moves through focus. While coherent illumination $(\sigma \approx 0)$ produces high contrast peaks over a large focal range, partially coherent illumination produces a characteristic series of diminishing peaks that can reveal the $\sigma$ value by comparison with modeling.

We recorded several such series using large, dense-line patterns with 240, 320, and 400-nm line widths, moving as far as $30 \mu \mathrm{m}$ out of focus in both directions. The 0.0875-NA zoneplate lens was used for these measurements. For comparison with the experimental data, calculations were performed using the same wavelength, distances, NA, and mask pattern, and a range of $\sigma_{x}$ values chosen to capture the behavior of the angle-scanning horizontal KB mirror. Knowing that the illumination angles are static in the $y$-direction, we used the approximation that $\sigma_{y}=0$. Data from measurements and modeling are shown in Fig. 7. For 320 and 400-nm line widths, the experimental data is compared with two model $\sigma_{x}$ values, as indicated. In each case, a scaling factor has been applied to the model data to match the experimental contrast at best focus. (The model value would otherwise be unity at best focus.) Figure 7c contains five image details, approximately $2-\mu \mathrm{m}$ wide, that have been extracted form near the center of several images in the through-focus series.

The modeling comparisons show that $\sigma_{x}$ values close to 0.125 are a good approximation for the coherence properties we observe. Discrepancies between the measured data and the model likely come from aberrations in the zoneplate lens and its alignment, and from imperfect $z$ actuation over the large through-focus distance.

It is important to note that the partial coherence scales inversely with the imaging lens' NA. Since the measurements presented here were made using the 0.0875 -NA lens, $\sigma_{x}$ will be higher when a lower-NA zoneplate is used and all other conditions are the same. For example, with the 0.075 or $0.0625-\mathrm{NA}$ lenses, $\sigma_{x}$ should grow to 0.146 and 0.175 , respectively. 


\section{CONCLUSION}

Recent upgrades to the the SEMATECH Berkeley Actinic Inspection Tool have improved its performance in the areas of aerial image resolution, illumination uniformity, and partial coherence. Using a variety of new, high-NA zoneplate lenses, the AIT emulates the resolution of EUV lithography tools with NA values up to 0.35 . The AIT provides above $90 \%$ uniformity for $2-\mu \mathrm{m}$ square (mask) area, and above $87 \%$ for a $3-\mu \mathrm{m}$ area. Dense-line contrast measurements through focus were compared with modeling to show that the $x$-direction partial coherence, $\sigma_{x}$, is approximately 0.125 for the $0.35-\mathrm{NA} 4 \times$ equivalent ( 0.0875 NA) lens. Based on that measurement, we predict that $\sigma_{x}$ is 0.175 for the 0.25 -NA $4 \times$ equivalent $(0.0625 \mathrm{NA})$ lens.

\section{ACKNOWLEDGMENTS}

The authors gratefully acknowledge the support of SEMATECH, including Stefan Wurm, Kim Dean, and others who worked to ensure the ongoing success of this project. LBNL team members who have been instrumental in the development of the AIT include Paul Denham, Rene Delano, Brian Hoef, and Kevin Bradley. The authors would also like to thank Bruno LaFontaine of AMD, and Ted Liang of Intel, and Pat Kearney of SEMATECH for providing the masks used in this research. This work is funded by SEMATECH under Project LITH-343S2, and was performed under the auspices of the U.S. Department of Energy by University of California Lawrence Berkeley National Laboratory.

\section{REFERENCES}

1. P. P. Naulleau, C. N. Anderson, J. Chiu, K. Dean, P. Denham, K. A. Goldberg, B. Hoef, S. Huh, G. Jones, B. LaFontaine, A. Ma, D. Niakoula, J. Park, T. Wallow, Proc. SPIE 6921, 69213N (2008).

2. K. A. Goldberg, A. Barty, Y. Liu, P. Kearney, Y. Tezuka, T. Terasawa, J. S. Taylor, H.-S. Han, and O. R. Wood II., J. Vac. Sci. \& Technol. B 24 (6), 2824 (2006).

3. K. A. Goldberg, P. P. Naulleau, A. Barty, S. B. Rekawa, C. D. Kemp, R. F. Gunion, F. Salmassi, E. M. Gullikson, E. H. Anderson, H.-S. Han, Proc. SPIE 6730, 67305E (2007). 
4. K. A. Goldberg, S. B. Rekawa, C. D. Kemp, A. Barty, E. H. Anderson, P. Kearney, H.-S. Han, Proc. SPIE 6921, 69213U (2008).

5. G.-S. Yoon, H. Kim, H.-J. Oh, H.-S. Kim, H.-S. Sim, S.-H. Lee, G.-B. Kim, S.-M. Huh, K. A. Goldberg, A. Barty, S.-S. Kim, H.-K. Cho, EUVL Symposium 2007, Sapporo, Japan October 31, 2007.

6. W. Cho, H.-S. Han, K. A. Goldberg, P. A. Kearney, C.-U. Jeon, Proc. SPIE 6730, 673013 (2007).

7. H.-S. Han, W. Cho, K. A. Goldberg, E. M. Gullikson, C.-U. Jeon, S. Wurm, Proc. SPIE 6921, 62911Y (2008). 
Fig. 1. A new zoneplate array design allows one of five different zoneplates to be selected. Zoneplates are held within $1 \mathrm{~mm}$ above the mask. The illuminating beam passes downward through the un-patterned portion of the $\mathrm{Si}_{3} \mathrm{~N}_{4}$ membrane. Reflected light is collected by one of the off-axis zoneplates and focused on the CCD. Two small zoneplates on the ends serve as distinct alignment features.

Fig. 2. Image details from 100-nm line-width elbow patterns, recorded (a) before and (b,c) after the AIT upgrades. Smaller CCD pixels and higher magnification zoneplate lenses result in higher pixel density. Each square in the checker patterns is five CCD pixels wide in the raw data. Regions near the bottom of the images were averaged vertically to produce the profiles. Fig. 3. Illumination uniformity calculated from image sub-regions of a blank mask. Details corresponding to $12-\mu \mathrm{m}$-wide mask regions with (a) static and (b) scanning illumination. As shown schematically (c), the minimum and maximum intensity values found within 100 different adjacent or overlapping regions near the center of the CCD are used to compute the average and standard deviation (d) for a range of mask-region widths.

Fig. 4 Darkfield CTF computed from elbow patterns with mask line widths between 100 and 500-nm. In each case the highest measured contrast from four available elbow directions is shown. The dashed curves show the theoretical performance calculated with $\sigma_{y}=0$, and $\sigma_{x}=$ 0.125 and 0.175 for the 0.0625 -NA and 0.0875 -NA cases, respectively.

Fig. 5. Measured brightfield (BF) and darkfield (DF) CTF data from the 0.0875 NA zoneplate.

Fig. 6. Contours and image details from a through-focus series of a single, 175-nm square contact. Images are individually scaled. The contours levels are drawn in 0.125 steps, normalized to the peak intensity of the series. The relative focal displacement $(z)$ and scaled peak magnitude values are given.

Fig. 7. The illumination partial coherence is determined by studying dense-line contrast through focus. Experimental data (open symbols) is compared with models (dashed lines) having different $\sigma_{x}$ values. (a) 320-nm and (b) 400-nm lines provide consistent results showing that $\sigma_{x}$ is close to 0.125 for the $0.0875 \mathrm{NA}(0.35-\mathrm{NA} 4 \times$ equivalent) lens. (c) Two- $\mu \mathrm{m}$-square image details from the through-focus series are shown. Contrast calculations are based extracted image sub-domains, like this. 


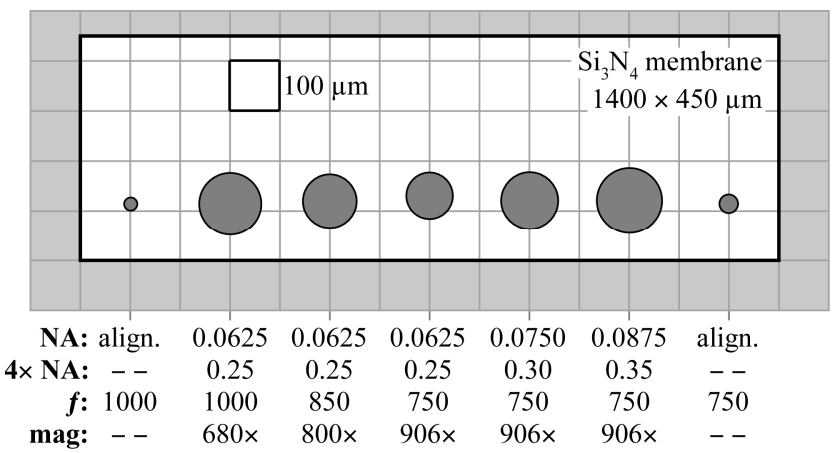

Electronic version is available

Fig 1. 


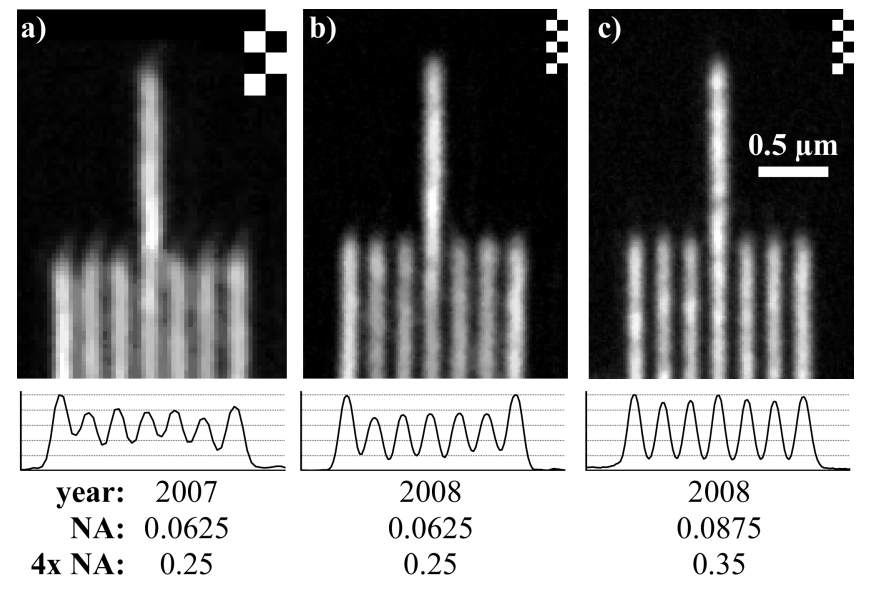

Electronic version is available

Fig. 2. 
a)

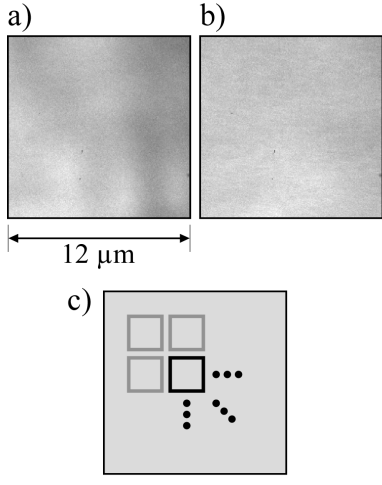

d) illumination uniformity

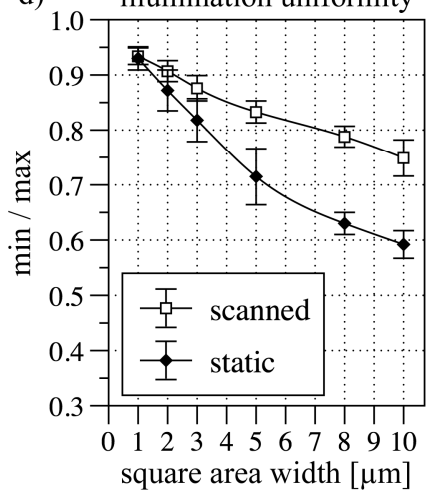

Electronic version is available

Fig. 3. 


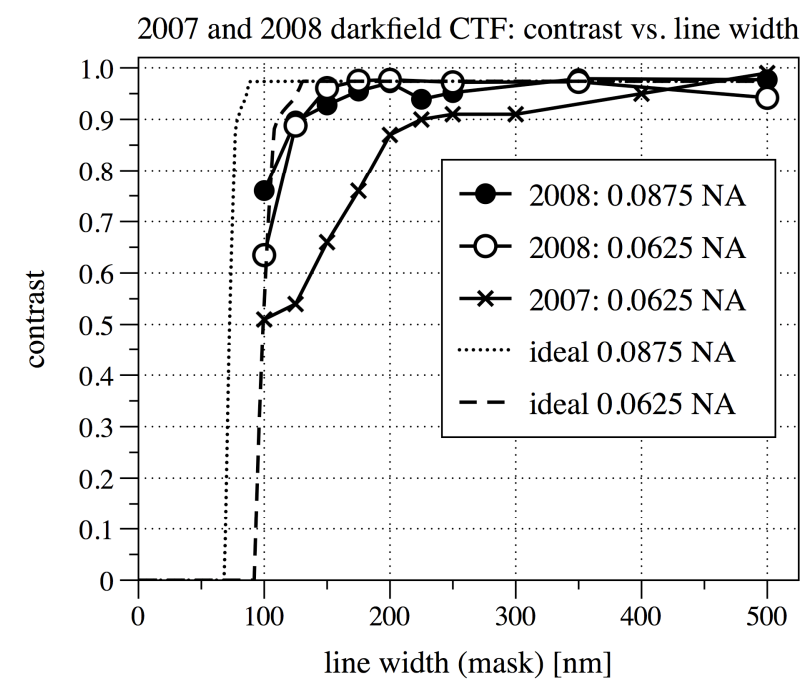

Electronic version is available

Fig. 4. 


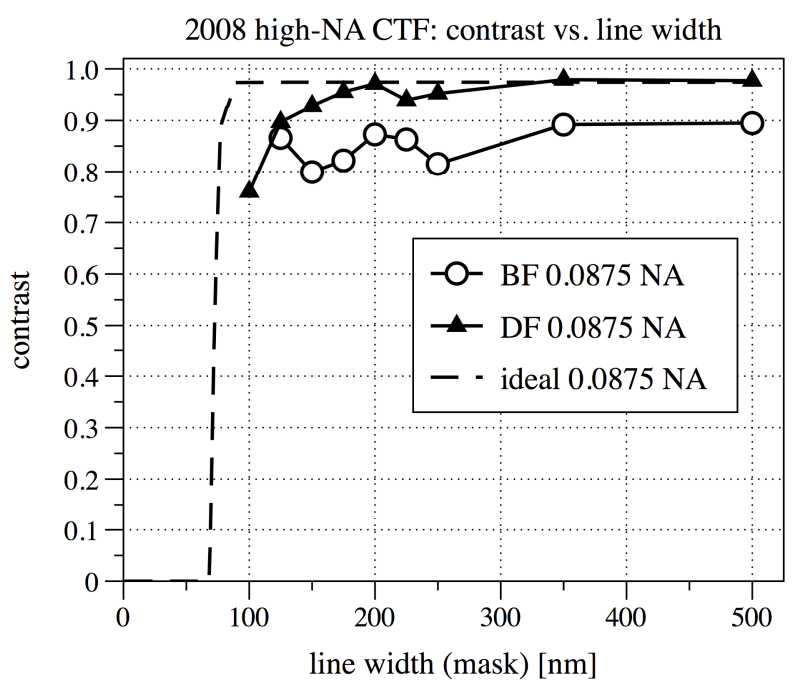

Electronic version is available

Fig. 5. 


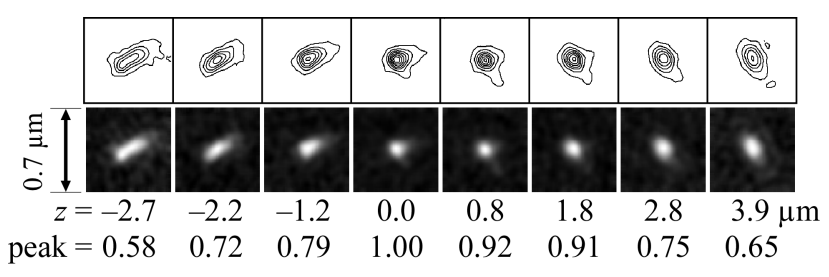

Electronic version is available

Fig. 6. 
a)

320-nm lines: contrast through focus

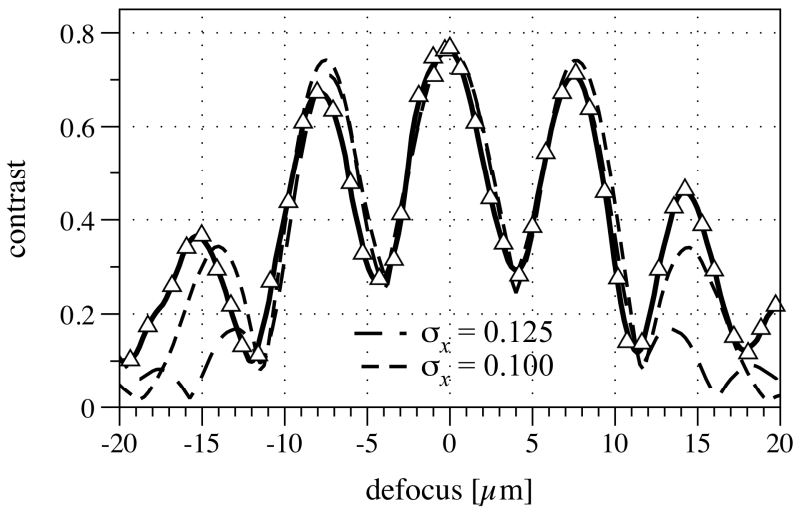

b)
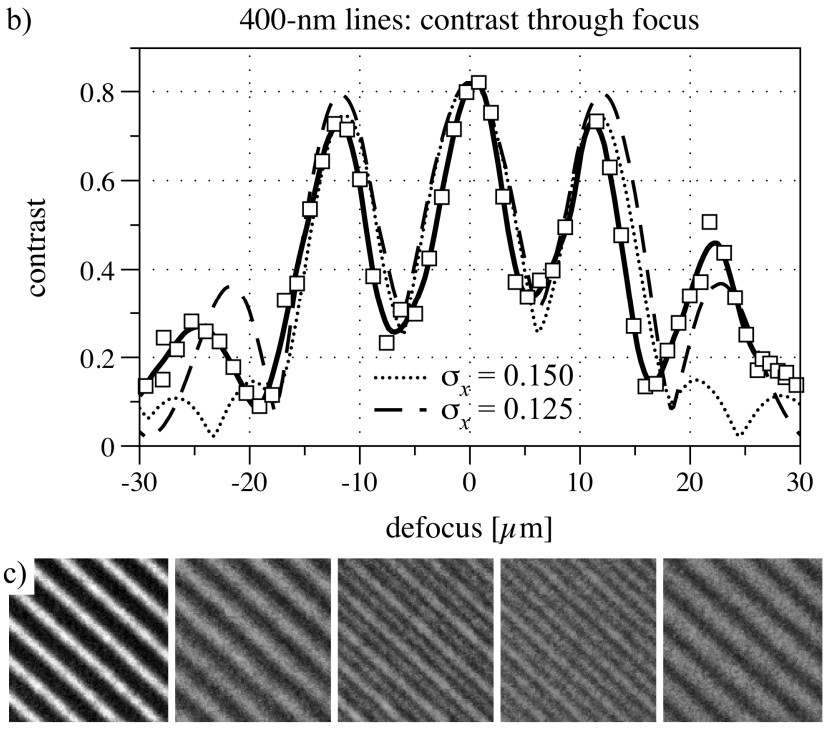

Electronic version is available

Fig. 7.

This work was supported by the Director, Office of Science, Office of Basic Energy Sciences, of the U. S. Department of Energy under Contract No. DE-AC02-05CH11231 